\title{
Multicolor CCD photometry of the open cluster NGC 752
}

Stanislava Bartašiūtè, ${ }^{1}$ Robert Janusz, ${ }^{2,3}$ Richard P. Boyle, ${ }^{3}$ A. G. Davis Philip ${ }^{4}$ and Viktoras Deveikis ${ }^{1}$

\author{
${ }^{1}$ Astronomical Observatory of Vilnius University, Čiurlionio 29, LT-03100, Vilnius, Lithuania \\ email: stanislava.bartasiute@ff.vu.lt \\ ${ }^{2}$ University School Ignatianum, ul. Kopernika 26, 31-501, Kraków, Poland \\ email: rj@jezuici.krakow.pl \\ ${ }^{3}$ Vatican Observatory Research Group, Steward Observatory, Tucson, AZ 85721, USA \\ email: boyle@ricci.as.arizona.edu \\ ${ }^{4}$ ISO and Union College, 1125 Oxford Place, Schenectady, NY 12308, USA \\ email: agdp@union.edu
}

\begin{abstract}
We obtained CCD observations of the open cluster NGC 752 with the $1.8 \mathrm{~m}$ Vatican Advanced Technology Telescope (Mt. Graham, Arizona) with a 4K CCD camera and eight intermediate-band filters of the Stromvil (Strömgren + Vilnius) system. Four $12^{\prime} \times 12^{\prime}$ fields were observed, covering the central part of the cluster. The good-quality multicolor data made it possible to obtain precise estimates of distance moduli, metallicity and foreground reddening for individual stars down to the limiting magnitude, $V=17.5$, enabling photometric identification of faint cluster members. The new observations provide an extension of the lower main sequence to three magnitudes beyond the previous (photographic) limit. A relatively small number of photometric members identified at fainter magnitudes seems to be indicative of actual dissolution of the cluster from the low-mass end.
\end{abstract}

Keywords. techniques: photometric, open clusters and associations: individual (NGC 752)

\section{Introduction}

The Galactic open cluster NGC $752\left[\alpha(2000)=01^{\mathrm{h}} 57.7^{\mathrm{m}}, \delta(2000)=+37^{\circ} 47^{\prime} ; \ell=\right.$ $137.1^{\circ}, b=-23.3^{\circ}$, at a distance of $450 \mathrm{pc}$ from the Sun, is one of the very few clusters in the solar neighborhood with an age between 1 and 2 Gyr. With its relatively small number of stars $(\sim 130)$, which are observed out to $40^{\prime}$ from the cluster center, NGC 752 has been the subject of some controversy regarding its lower main sequence: the great majority of the stars are found in the region of the turnoff, whereas the lower part of the main sequence appears to be very sparsely populated. From the available photometric, proper-motion and radial-velocity data the cluster's main sequence is known at best to $M_{V} \sim+6$ mag. This may be due to both a lack of observations at magnitudes fainter than the present (photographic) limit and dynamic escape of low-mass stars from the cluster. To define the extension of the cluster's main sequence towards fainter magnitudes, we obtained CCD observations of several $12^{\prime} \times 12^{\prime}$ fields in the central part of the cluster.

\section{Observations and data reductions}

Observations were taken in November 2007 and 2008 at the $1.8 \mathrm{~m}$ Vatican Advanced Technology Telescope (VATT) on Mt. Graham, Arizona, with a 4K CCD camera and eight intermediate-band filters of the Stromvil (Strömgren + Vilnius) system. The scale 


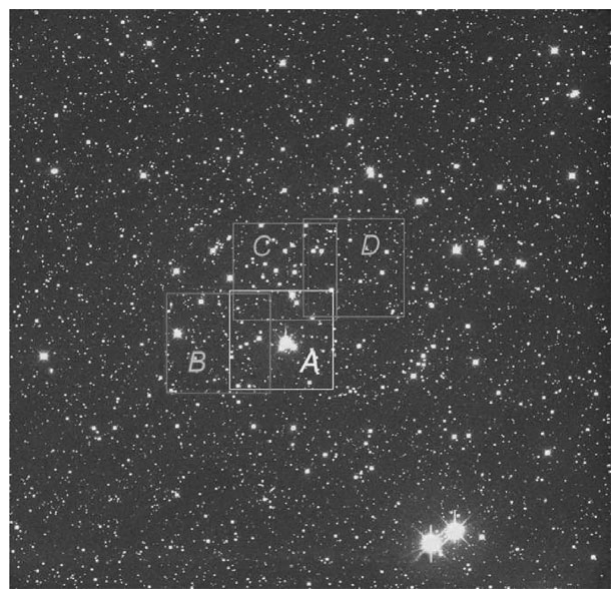

Figure 1. NGC 752. Marked are four VATT $4 \mathrm{~K}$ CCD fields of $12^{\prime} \times 12^{\prime}$ each.

on the chip is $0.37^{\prime \prime}$ per pixel, yielding a field of view of $12^{\prime} \times 12^{\prime}$. Both long and short exposures were obtained in four slightly overlapping fields, A, B, C and D (Figure 1).

CCD frames were processed using the CommandPнот software (Janusz 2007). Flatfield corrections and color transformations were derived from observations of the standard cluster M67. Photoelectric standards in the field of NGC 752 (Bartašiūtè et al. 2007) were also used, either for calibrations or as a check on the external accuracy of our photometry. A total of 440 stars down to $18.0 \mathrm{mag}$ have been measured in the four fields (Figure 2).

\section{Results}

To identify photometric members of the cluster, we determined for individual stars the absolute magnitudes, $M_{V}$, interstellar reddening values, $E(Y-V)$, and metallicities, $[\mathrm{Fe} / \mathrm{H}]$. Accuracies of $\pm 0.2 \mathrm{mag}, \pm 0.02 \mathrm{mag}$ and \pm 0.15 dex for these three parameters, respectively, were regularly obtained for stars brighter than magnitude 16.5.

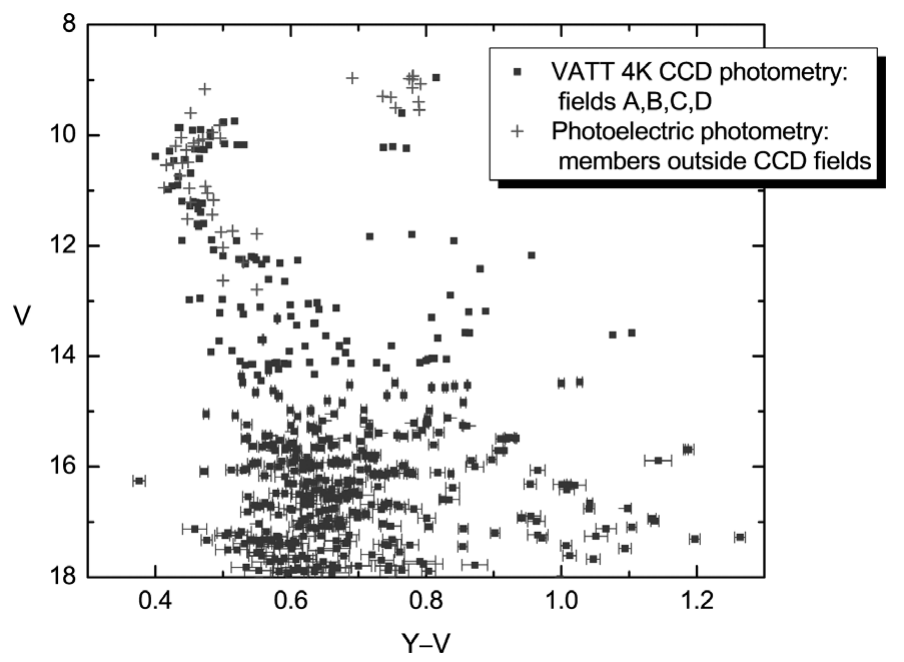

Figure 2. $(V, Y-V)$ diagram for stars in four CCD fields (solid squares). The cluster members outside these fields, with photoelectric Vilnius photometry, are also plotted (crosses). 
Photometric members were selected according to the values of the cluster parameters determined earlier from photoelectric Vilnius photometry of 62 cluster stars (Bartašiūtè et al. 2007)-(m-M $)_{V}=8.38 \pm 0.14 \mathrm{mag}, E(Y-V)=+0.03 \pm 0.01 \mathrm{mag}$ and $[\mathrm{Fe} / \mathrm{H}]=-0.11 \pm 0.08$ dex - by adopting the $3 \sigma$ criterion to allow for the effect of classification errors and taking into account the photometric effect of unknown binaries.

Seventy of the 440 stars classified have cluster membership status known from propermotion and/or radial-velocity data (Daniel et al. 1994). Therefore, we were able to check the validity of our photometric membership estimates. For 65 such stars, photometric and astrometric results show a remarkable coincidence, and only five stars, or less than $10 \%$, show the opposite. The overwhelming majority of background stars (the crowding below $V \approx 15 \mathrm{mag}$ in the the diagram of Figure 2) are classified as dwarfs of spectral types from late $\mathrm{F}$ to early $\mathrm{K}$, having, in most cases, a mild metal deficiency.

The photometric members are shown in Figure 3, together with the best-fitting isochrone (1.5 Gyr) and model binary trajectories. The new observations provide an extension of the lower main sequence to four magnitudes beyond the photoelectric limit and to three magnitudes beyond the proper-motion (photographic) limit. The color-magnitude diagram (CMD) clearly shows an inadequate number of low-mass stars with respect to the expected main sequence, at least in the central, observed part of the cluster. In this context, it is interesting to note that the red giants in the cluster, regardless of their higher initial masses, seem to be amongst the least centrally concentrated cluster stars.

Within the cluster radius, the existing data give a more or less complete census of the member stars only for the upper part of the CMD. Therefore, we can make only a cursory comparison of the number of observed stars with that following from the cluster's initial mass (Figure 4). To obtain the mass of a cluster containing, like NGC 752, 15 red giant stars observed at an age of 1.5 Gyr, we assumed a Kroupa (2001) stellar initial mass function (IMF) and generated with the SIMCLUST tools (Deveikis et al. 2008) 1000 artificial clusters in the mass interval from 1000 to $5000 \mathrm{M}_{\odot}$. Our simulations, which included stellar evolution for single stars, give an initial mass of $2200 \mathrm{M}_{\odot}$. If we extrapolate the number of identified photometric members across the entire diameter of

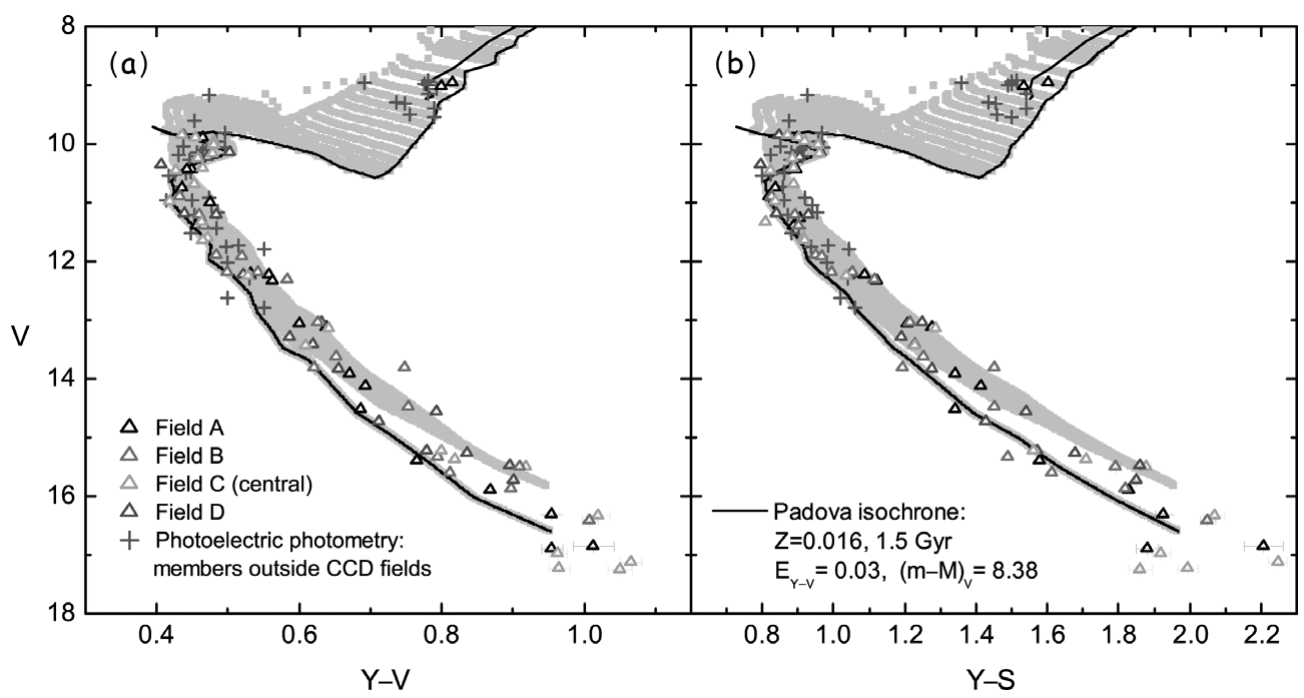

Figure 3. CMDs of the photometric members of NGC 752. The shaded area denotes model binary trajectories outlined by the best-fitting isochrone. 


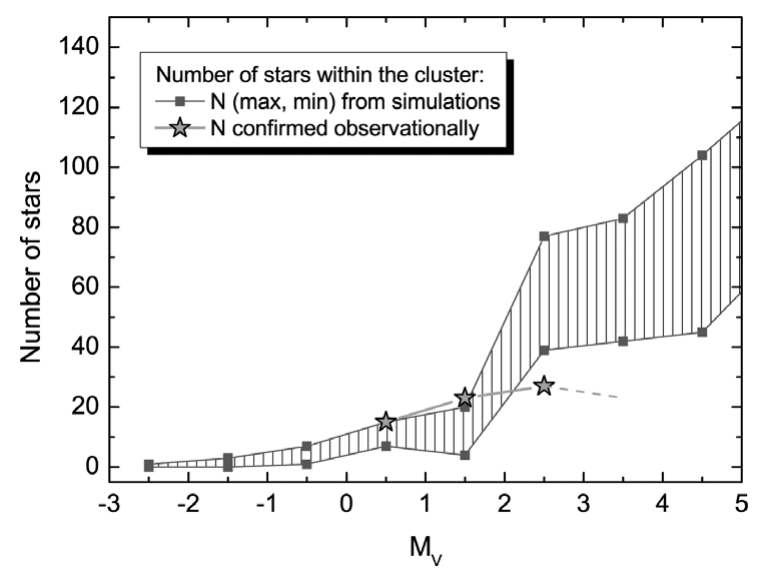

Figure 4. Present luminosity function based on Monte Carlo simulations.

the cluster, we would still have a fairly small number of lower-mass stars relative to the initial mass spectrum.

According to the predicted dissolution times for clusters in the solar neighborhood given by Lamers \& Gieles (2006), the survival time of a cluster with an initial mass of $2200 \mathrm{M}_{\odot}$ should be $0.6 \mathrm{Gyr}$, i.e., much shorter than the present age of NGC 752 . The discrepancy can be reconciled by assuming for this cluster a larger initial mass. As Figure 4 suggests (see the observed number of stars in the range $0<M_{V}<2$ mag), the cluster mass is likely underestimated. There are at least two reasons for such a possibility. First, the actual number of red giants, on which the basic point of our simulations rests, can be incomplete. Indeed, a radial-velocity survey by Mermilliod et al. (1998) has revealed an additional two red giants at nearly $100^{\prime}$ from the cluster center, suspected to be corona or escaping members. Second, the result is highly dependent on the assumed stellar IMF: assuming a Salpeter IMF, the initial mass of the cluster should be twice that simulated with a Kroupa-type IMF.

In summary, we confirm the status of NGC 752 as a dissolving cluster. To discern the escaping low-mass members, high-precision photometric, astrometric and radial-velocity observations secured in the outer parts of the cluster would be most helpful.

\section{References}

Bartašiūtè, S., Deveikis, V., Straižys, V., \& Bogdanovičius, A. 2007, BaltA, 16, 199

Daniel, S. A., Latham, D. W., Mathieu, R. D., \& Twarog, B. A. 1994, PASP, 106, 281

Deveikis, V., Narbutis, D., Stonkutè, R., Bridžius, A., \& Vansevičius, V. 2008, BaltA, 17, 351

Janusz, R. 2007, BaltA, 16, 467

Kroupa, P. 2001, MNRAS, 322, 231

Lamers, H. J. G. L. M. \& Gieles, M. 2006, A\&A (Letters), 455, L17

Mermilliod, J.-C., Mathieu, R. D., Latham, D. W., \& Mayor, M. 1998, A\&\&A, 339, 423 